\title{
Optimization design of Close-top Mill Housing Based on Agent Model and NSGA-II Algorithm
}

\author{
Yanjun $\mathrm{Shi}^{1}$, Xianchao Wang $^{1}$, Xiaojun Zheng ${ }^{2}$ \\ ${ }^{1}$ School of Mechanical Engineering, Dalian University of Technology, Dalian, 116024, China. \\ ${ }^{2}$ Key Laboratory of Advanced Design and Intelligent Computing, Ministry of Education of Dalian \\ University, Dalian 116622, China
}

Keywords: EBF-NN, RBF-NN, Kriging, NSGA-II, Mill housing, Surrogate models.

\begin{abstract}
Conventional engineering optimization design technology is often computational expense and highly time cost, thus we need to take approximation technique to speed up the design process and reduce design cost. We herein would introduce the close-top mill housing optimization model, take Optimal Latin Hypercube Sampling (OLHS) experimental design strategies to determine the sample, analyse the difference among EBF, RBF, Kriging surrogate model, introduce NSGA-II algorithm briefly and study the performance of method based on surrogate model and optimization algorithm.
\end{abstract}

\section{Introduction}

Generally numerical simulation system cost too much and may fail in obtaining the optimal solution in complicated engineering design optimization problems. The optimization method based on approximate model is of good global performance and could effectively save computing resources. Sun took the maximum longitudinal-stiffness as the goal and use an optimization method based on three-dimensional finite element analysis and FEM software to design the main parameters of the mill housing[1]. Lei proposed the optimization strategy using dynamic radial basis function agent model to test the NASA speed reducer optimization design, which make the time reduce by 50\%[2]. MAK applied the smaller size EBF networks with basis function parameters determined by the EM algorithm to the speaker verification and outperformed the large RBF networks trained in the conventional approach[3]. Wang optimized the aerodynamic design of aerofoil by using the kriging model, which increased the lift-drag ratio of aerofoil by $81 \%$ with the cross section area of the aerofoil only decreasing less than $1 \%$ and greatly save the calculating resources[4].

In this paper, firstly we introduce the close-top mill housing optimization model, the constraint conditions and optimization objectives. Then we take OLHS experimental design strategies to determine the sample space and test sample. Further, we describe and compare the difference of EBF, RBF and Kriging surrogate model and explain the NSGA-II algorithm briefly. Finally, we study the performance of method based on surrogate model and optimization algorithm by comparing and analysing prediction results and optimization results.

\section{Description of close-top mill housing optimization model}

The close-top mill housing is symmetrical structure and its two-dimensional and threedimensional map are shown in Figure 1. The size and scope of the design variables and the objectives have been listed in Table 1 and Table 3, and the remaining fixed dimensions are $\mathrm{BW}=4050, \mathrm{H} 6=5000, \mathrm{H} 3=400, \mathrm{H} 5=180, \mathrm{~K} 1=800, \mathrm{~B} 2=1510, \mathrm{R} 3=350, \mathrm{R} 4=225, \mathrm{R} 6=25$, $\mathrm{R} 1=50, \mathrm{R} 2=50, \mathrm{R} 7=200[1]$.

We would take $1 / 4$ of the structure to build and analyze the mode and apply symmetry constraints in A, C plane. In the rolling process, forces that frame bears are very complex, in which the rolling force is maximum. So we take the rolling force as the external load (20.271Mpa on the above beam, 9.0774Mpa on the below beam) and imposed rigid constraints at the bolt of the close- 
top mill housing. In order to eliminate the calculation error of the results caused by structural model, we would enter the original values of the mill housing and the optimal values analyzed by Ansys into the model to calculate the responding results.

Table 1: range of the independent variables and target variables

\begin{tabular}{lll}
\hline $\begin{array}{l}\text { Independent } \\
\text { variables/mm }\end{array}$ & $\begin{array}{l}\text { Independent } \\
\text { variables } / \mathrm{mm}\end{array}$ & $\begin{array}{l}\text { Constrained and } \\
\text { Target variables }\end{array}$ \\
\hline $720 \leq \mathrm{B} 1 \leq 850$ & $50 \leq \mathrm{R} 1 \mathrm{~W} \leq 770$ & Misses $\leq 50 \mathrm{Mpa}$ \\
$900 \leq \mathrm{H} 1 \leq 1200$ & $50 \leq \mathrm{R} 2 \mathrm{~W} \leq 770$ & Volume $\leq$ Vinitial \\
$900 \leq \mathrm{H} 2 \leq 1100$ & $600 \leq \mathrm{T} \leq 700$ & $\mathrm{dx} \leq$ dxinitial \\
$0 \leq \mathrm{H} 4 \leq 200$ & $600 \leq \mathrm{R} 5 \leq 700$ & dy $\leq$ dyinitial \\
\hline
\end{tabular}
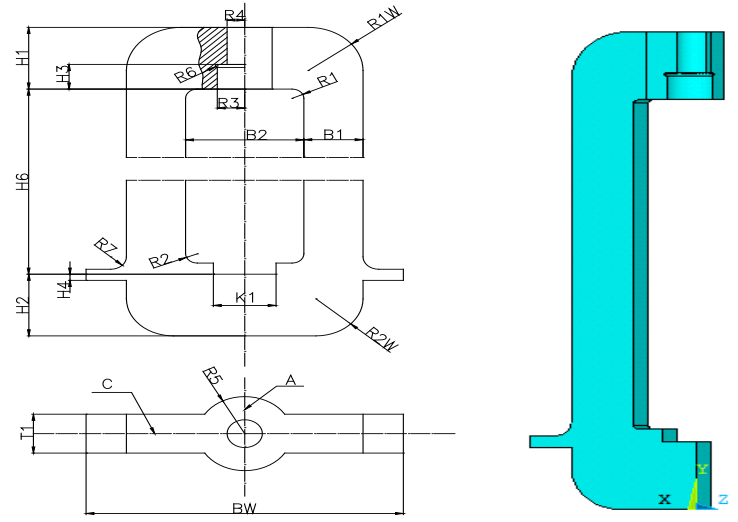

Fig.1: structure diagram of close-top mill housing

\section{Design of Experiment}

\subsection{Sample Points}

Latin Hypercube Sampling (LHS) method is a multidimensional hierarchical sampling plan. Meanwhile randomness of the combination among design variables leads LHS outcome uncertain and make it difficult to guarantee the prediction accuracy of the surrogate model[5]. Therefore, Optimal Latin Hypercube Sampling (OLHS) would be used to optimize sample points on the specific location, which could greatly ameliorate uniformity of Random Latin hypercube Sampling and make fit more precisely. Initialization of the approximation model with OLHS method often requires $2 n+1$ design points at least, in which $n$ is the number of inputs, and we would take OLHS experimental design strategies to determine the 40 sample points and 10 error analysis test sample points.

\subsection{Error Analysis}

Since the units and dimension of response values of different variables is different, root mean square error fail in expressing the relative quality of different models. For the purpose of testing and evaluating the performance of different surrogate model accurately, we use multiple correlation coefficients(R-square) to evaluate approximate model. When the value of $\mathrm{R}$ square is closer to 1 , the fitting effect will be better.

$$
R^{2}=1-\frac{\sum_{k=1}^{m}\left(\hat{y}\left(x^{k}\right)-\bar{y}\left(x^{k}\right)\right)^{2}}{\sum_{k=1}^{m}\left(y\left(x^{k}\right)-\bar{y}\left(x^{k}\right)\right)^{2}} \quad\left(\mathrm{R}^{2} \leq 1\right) \quad \text { (1) } \quad \text { In (1), } y\left(x^{k}\right) \text { and }
$$

$\hat{y}\left(x^{k}\right)$ are the actual response and the predictive value in the $k$ th test sample points $x^{k}, \bar{y}\left(x^{k}\right)$ is the mean value of actual response, $m$ is the number of test sample points. 


\section{Surrogate Model and NSGA-II Algorithm}

Surrogate models predictive technology has become a fast and effective way to solve complex engineering problems, and RBF-NN, EBF-NN and Kriging are of particular significance. When RBF and EBF choose Gaussian function as basis function, the three methods look similar and we will compare and analyse the differences between them.

\subsection{RBF-NN}

RBF-NN has outstanding performance in reasonably fast training and reasonably compact networks. They are effective in approximating a wide range of nonlinear spaces, but requires more neurons in hidden layer network and is likely to over-fit the model[2, 6]. The independent variable is the Euclidean distance between test points and the sample points in RBF-NN.

$$
\hat{f}(x)=\sum_{j=1}^{m} w_{j} \exp \left(-\theta_{j}\left(x_{j}-c^{(j)}\right)^{2}\right)
$$

In (2), ${ }^{x_{j}}$ is the $j$ th input vector, $c^{(j)}$ is the mean vector of the $m$ th basis function, ${ }^{w_{j}}$ and $\theta_{j}$ are the parameters to estimate.

\subsection{EBF-NN}

Elliptical Basis Function Neural Network (EBF-NN) is often regarded as an extension of Radial Basis Function Neural Network (RBF-NN)[3]. The independent variable is the Mahalanobis distance between test point and the sample point, thus there exist more adjustable parameters to improve the accuracy of the model in EBF-NN.

$$
\hat{f}(x)=\sum_{j=1}^{m} w_{j} \exp \left(-\theta_{j}\left(x_{j}-c^{(j)}\right)^{T} \sum_{m}^{-1}\left(x_{j}-c^{(j)}\right)\right)
$$

In (3), $\sum_{m}^{-1}$ is the covariance matrix of the $m t h$ basis function, others are same with formula 2.

\subsection{Kriging}

Kriging surrogate model is based on variogram theory and structure analysis, and is also a method to make an unbiased optimal estimation for the regionalized variables within a limited area[4, 7]. Correlation function of kriging adopt Gaussian function and allow the exponent $\left(P_{j}=\left\{p_{1}, p_{2}, \cdots p_{k}\right\}^{T}\right)$ to vary (typically $\left.p_{j} \in[1,2]\right)$ for each dimension.

$$
\operatorname{Cov}\left[Y\left(x^{(i)}\right), Y\left(x^{(l)}\right)\right]=\exp \left(-\sum_{j=1}^{k} \theta_{j}\left(x_{j}^{(i)}-x_{j}^{(l)}\right)^{\mathrm{p}_{j}}\right)
$$

In (4), $\mathrm{j}$ is the number of known design variables, and ${ }^{x_{j}^{(i)}}, x_{j}^{(l)}$ represents the $i t h$, and the Ith samples of $j$ th variable respectively, $\theta_{j}, p_{j}$ are the parameters to be estimated.

\subsection{NSGA-II}

In the Non-dominated Sorting Genetic Algorithm (NSGA-II), each objective is treated separately and a pareto front is constructed by selecting feasible non-dominated designs[8]. Selection process is based on two main mechanisms, "non-dominated sorting" and "crowding distance sorting". By the end of the optimization a pareto set is constructed where each design has the best combination of objective values and improving one objective is impossible without sacrificing one or more of the other objectives.

In summary, the optimization steps of close-top mill housing based on EBF-NN, RBF-NN, Kriging Agent model and NSGA-II algorithm are shown in Fig.2.

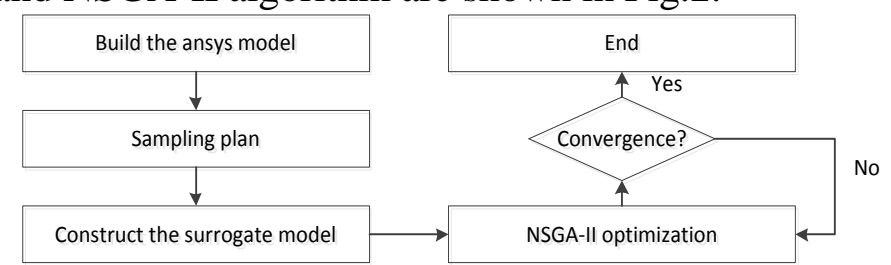

Fig.2: Flowchart for optimization process of Close-top Mill housing design 


\section{Experimental results and analysis}

\subsection{Prediction design}

We select 40 sample points for building the model and 10 sample points for error analysis with OLHS methods. After constructing the surrogate model, we could obtain the multiple correlation coefficients by calculating the predicted value and the actual value. The results are listed in Table 2 . With the same number of sample points, three methods all have guaranteed high prediction accuracy except for misses and the prediction accuracy with RBF-NN method is overall higher than that with EBF-NN and kriging method.

Compared to the RBF-NN surrogate model, EBF-NN and kriging surrogate model have more parameters to adjust the approximation model, which mean they own the ability to fit the model more accurately and need more sampling points to compute the parameters as well. Though we can improve the prediction accuracy of EBF and kriging surrogate model by increasing the sampling points, reducing the number of experiments is of significant to solve practical engineering problems in the case of meeting the accuracy. Considering the ceiling for misses is relatively high, we didn't increase the sampling points to improve its prediction accuracy especially.

Table 2: R-square Analysis Comparing

\begin{tabular}{llllc}
\hline Algorithm & $\mathrm{dx}$ & $\mathrm{dy}$ & misses & volume \\
\hline RBF-NN & 0.99489 & 0.99865 & 0.63778 & 0.99953 \\
EBF-NN & 0.99399 & 0.99833 & 0.65903 & 0.99908 \\
Kriging & 0.98396 & 0.98634 & 0.54745 & 0.99451 \\
\hline
\end{tabular}

\subsection{Optimization design}

We take the quality of the close-top mill housing as the target of optimization (longitudinal deformation "dy", transversal deformation "dx"), and consider the weight and stress as constraint conditions (volume "volume", stress "misses"). The optimization process and optimization results are shown in figure 3 and table 3. Compared with Ansys optimization results, dx decreases 5.0\%, dy decreases 3.7\%, misses increases 1.7\% and Volume increases 4.5\% in EBF-NN optimization results; $\mathrm{dx}$ increases $5.6 \%$, dy increases 3.8\%, misses decreases $9.6 \%$ and volume decreases $1.6 \%$ in RBFNN optimization results; most are similar with it except for $\mathrm{dx}$, which increases $2.9 \%$ in Kriging optimization results.

When only taking half the time Ansys used, calculation results of Kriging model is almost the same with it, the results of RBF-NN model tend to ensure the quality and the results of EBF-NN model tend to reduce weight. These show the feasibility and effectiveness of method based on the surrogate model and optimization algorithm. Considering the relationship between the target and the design variables of the model is relatively simple, gradient optimization method Ansys owns comes rapidly to find the optimal value (96 times). For the more complicated models, gradient optimization method may fail, trap in the local optimal point or cost too much time. Moreover, the method based on the surrogate model and optimization algorithm could obtain a better application, greatly reduce the amount of calculation and meet the requirement of engineering design.

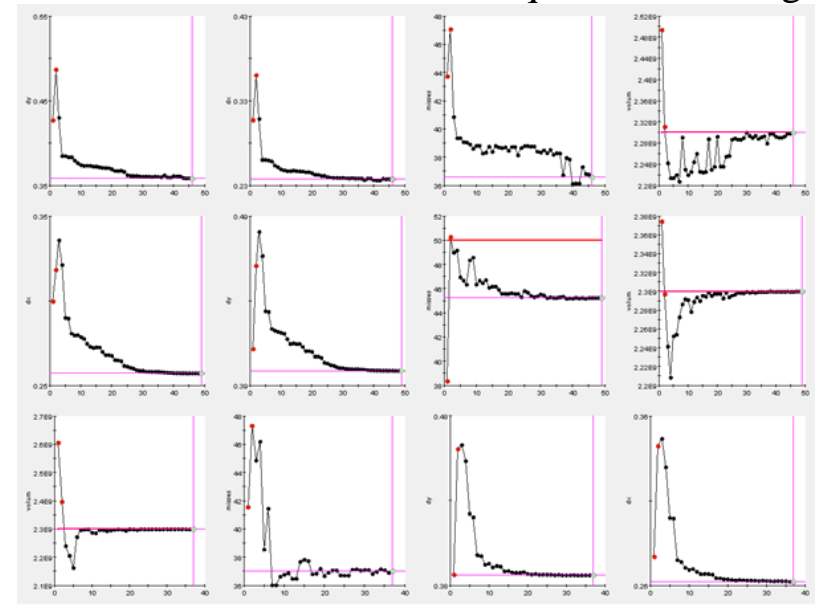

Fig.3: Optimization process of RBF, EBF, Kriging for dx, dy, misses, volume 
Table 3: Comparison among EBF-NN, RBF, Kriging, Ansys optimization result

\begin{tabular}{|c|c|c|c|c|c|}
\hline \multirow[t]{2}{*}{ Variable } & \multirow{2}{*}{$\begin{array}{l}\text { Initial } \\
\text { value }\end{array}$} & \multirow{2}{*}{\multicolumn{2}{|c|}{$\begin{array}{l}\text { Ansys } \\
\text { optimize }\end{array}$}} & \multicolumn{2}{|c|}{ RBF-Optimize } \\
\hline & & & & Predict & True \\
\hline $\mathrm{B} 1 / \mathrm{mm}$ & 760 & \multicolumn{2}{|l|}{720.26} & \multicolumn{2}{|c|}{720} \\
\hline $\mathrm{H} 1 / \mathrm{mm}$ & 1000 & \multicolumn{2}{|l|}{1167.7} & \multicolumn{2}{|c|}{1200} \\
\hline $\mathrm{H} 2 / \mathrm{mm}$ & 1000 & \multicolumn{2}{|l|}{1099.6} & \multicolumn{2}{|c|}{1100} \\
\hline $\mathrm{H} 4 / \mathrm{mm}$ & 100 & \multicolumn{2}{|l|}{0.83719} & \multicolumn{2}{|c|}{144} \\
\hline $\mathrm{R} 1 \mathrm{~W} / \mathrm{mm}$ & 700 & \multicolumn{2}{|l|}{768.13} & \multicolumn{2}{|c|}{769} \\
\hline $\mathrm{R} 2 \mathrm{~W} / \mathrm{mm}$ & 600 & \multicolumn{2}{|l|}{697.67} & \multicolumn{2}{|c|}{694} \\
\hline $\mathrm{R} 5 / \mathrm{mm}$ & 600 & \multicolumn{2}{|l|}{681.90} & \multicolumn{2}{|c|}{700} \\
\hline $\mathrm{T} 1 / \mathrm{mm}$ & 630 & \multicolumn{2}{|l|}{600.21} & \multicolumn{2}{|c|}{620} \\
\hline $\mathrm{Dx} / \mathrm{mm}$ & 0.3517 & \multicolumn{2}{|l|}{0.2533} & 0.2375 & 0.2406 \\
\hline $\mathrm{Dy} / \mathrm{mm}$ & 0.4721 & \multicolumn{2}{|l|}{0.3849} & 0.3580 & 0.3705 \\
\hline Misses/mpa & 44.78 & \multicolumn{2}{|l|}{37.83} & 36.57 & 38.47 \\
\hline Volum/mm3 & 2.186E9 & \multicolumn{2}{|l|}{ 2.191E9 } & 2.299E9 & 2.289E9 \\
\hline \multirow[t]{2}{*}{ Variable } & Initial & \multicolumn{2}{|c|}{ EBF-Optimize } & \multicolumn{2}{|c|}{ Kriging-Optimize } \\
\hline & value & Predict & True & Predict & True \\
\hline $\mathrm{B} 1 / \mathrm{mm}$ & 760 & \multicolumn{2}{|c|}{720} & \multicolumn{2}{|c|}{728} \\
\hline $\mathrm{H} 1 / \mathrm{mm}$ & 1000 & \multicolumn{2}{|c|}{1196} & \multicolumn{2}{|c|}{1198} \\
\hline $\mathrm{H} 2 / \mathrm{mm}$ & 1000 & \multicolumn{2}{|c|}{1010} & & \\
\hline $\mathrm{H} 4 / \mathrm{mm}$ & 100 & & & & \\
\hline $\mathrm{R} 1 \mathrm{~W} / \mathrm{mm}$ & 700 & & & & \\
\hline $\mathrm{R} 2 \mathrm{~W} / \mathrm{mm}$ & 600 & & & & \\
\hline $\mathrm{R} 5 / \mathrm{mm}$ & 600 & & & & \\
\hline $\mathrm{T} 1 / \mathrm{mm}$ & 630 & & & & \\
\hline $\mathrm{Dx} / \mathrm{mm}$ & 0.3517 & 0.2571 & 0.2675 & 0.2622 & 0.2606 \\
\hline Dy/mm & 0.4721 & 0.3987 & 0.3995 & 0.3860 & 0.3860 \\
\hline Misses/mpa & 44.78 & 45.24 & 34.21 & 37.01 & 37.83 \\
\hline Volum/mm3 & 2.186E9 & 2.300E9 & 2.155E9 & 2.299E9 & 2.191 E9 \\
\hline
\end{tabular}

\section{Conclusions}

When only spending half the time Ansys consumed, calculation results of Kriging model is almost the same with it, the results of RBF-NN model prefer to ensure the quality and the results of EBF-NN model prefer to reduce weight. For the more complicated models, the method could meet the requirement of engineering design and greatly reduce the amount of calculation. These all prove the feasibility and effectiveness of method based on the surrogate model and optimization algorithm and we could apply this method to solve more engineering problems.

\section{Acknowledgement}

This work was supported by the National Natural Science Foundation of China (Grant No. 61304206) and the Program of Liaoning Science and Research (Grant No. L2013460).

\section{References}

[1] Sun Zhan-Gang, Han Zhi-Ging, Wei Jian-Fang, Finite Element Analysis and Optimization Design of Close-top Mill Housing. Metallurgical Equipment, 145,pp. 8-11,2004.

[2] Peng Lei, Liu Li \& Long Teng, Optimization Strategy Using Dynamic Radial Basis Function Metamodel. Journal of Mechanical Engineering, 47(7),pp. 164-170, 2011.

[3] M. Mak, C. Li, \& X. Li, Maximum likelihood estimation of ellipitical basis function parameters with application to speaker verification. Proceedings of ICSP'98, pp. 1287-1290,1998. 
[4] Wang Xiao-Feng, Xi Guang, Aerodynamic Optimization Design for Airfoil on Kriging Model. Acta Aeronautica ET Astronautica Sinica, 26(5),pp. 545-549, 2005.

[5] X. Liao, Q. Li, X. Yang, W. Zhang \& W. Li, Multi-objective optimization for crash safety design of vehicles using stepwise regression model. Structural and multidisciplinary optimization, 35, pp. 561-569, 2008.

[6] M. J. Er, S. Wu, J. Lu \& H. L. Toh, Face recognition with radial basis function (RBF) neural networks. Neural Networks, IEEE Transactions on, 13(3), pp. 697-710, 2002.

[7] T. W. Simpson, Comparison of response surface and kriging models in the multidisciplinary design of an aerospike nozzle. 98,1998.

[8] K. Deb, A. Pratap, S. Agarwal \& T. Meyarivan, A Fast and Elitist Multiobjective Genetic Algorithm: NSGA-II. IEEE Transactions on Evolutionary Computation, IEEE Transactions on, 6(2), pp. 182-197, 2002. 